\title{
A complicação fístula faringocutânea após laringectomia total: uma análise preliminar
}

\author{
Complication in the pharyngeal cutaneous fistula after total laryngectomy: a \\ preliminary analysis
}

\author{
Mary Elizabeth de Santana ${ }^{*}$, Namie Okino Sawada ${ }^{2}$, Helena Megumi Sonobe ${ }^{3}$ e Márcia Maria Fontão Zago ${ }^{2}$
}

\begin{abstract}
Resumo
A complicação mais grave e freqüente apresentada pelo laringectomizado total é a fístula faringocutânea. Nesta investigação objetivou-se analisar a freqüência e os fatores de risco que contribuem para a formação de fístula faringocutânea, na literatura. Este é um estudo descritivo no qual analisaram-se 10 artigos indexados nos Bancos de Dados PUBMED e MEDLINE, que foram adquiridos na íntegra, no período de março de 2001 a março de 2002. Os fatores de risco identificados para a formação da fístula faringocutânea foram: radioterapia pré-operatória; dissecção radical do pescoço; tipo de material de sutura utilizado; traqueostomia pré-operatória; doenças sistêmicas; estadiamento do tumor; transfusão sangüínea no transoperatório; tipo de drenagem do pescoço; infecção da ferida e formação de hematoma. Esta complicação aumentou o período de internação e de cicatrização, com médias de 28 e de 36 dias, respectivamente. $\mathrm{O}$ tratamento predominante é ainda o conservador, no qual a enfermeira necessita de conhecimentos técnico-científicos, pois requer os cuidados intensivos, higiênicos e de tratamento da ferida. A habilidade de raciocínio clínico e técnica, aliada à busca de evidências e capacidade de relacionamento interpessoal da enfermeira, é que determinará a qualidade das intervençôes de enfermagem no atendimento das complicações do laringectomizado total.
\end{abstract}

Palavras-chave: laringectomia total; fístula faringocutânea; complicações; fatores de risco.

\section{Abstract}

The most severe and frequent complication presented by laryngectomized patients is the pharyngeal cutaneous fistula. This investigation aimed at analyzing the frequency in the literature of the risk factors that contribute to the formation of pharyngeal cutaneous fistula. This is a descriptive study in which authors analyzed 10 articles, indexed in the PUBMED and MEDLINE databases from March 2001 to March 2002. The risk factors for the formation of pharyngeal cutaneous fistula were: preoperatory radiotherapy; neck dissection, type of material used for sutura; preoperatory tracheostomy; systemic diseases; blood transfusion; neck draining type; infection and hematoma formation. This complication increases the hospitalization and cicatrization period, with an average of 28 days and 36 days, respectively. The predominant treatment is still conservative, in which the nurse needs technical and scientific knowledge as the care involves intensive and hygienic procedures and the wound treatment. The ability for clinical and technical reasoning linked to the search for evidences as well as nurse's capability of interpersonal relationship will determine the quality of nursing interventions in the care provided to the laryngectomized patient. Key words: total laryngectomy; pharyngeal cutaneous fistula; complications; risk factors.

\footnotetext{
${ }^{1}$ Doutoranda da EERP-USP, (Enfermagem Fundamental),Centro Colaborador da OMS para o Desenvolvimento de Pesquisa em Enfermagem.

Professor Assistente da UFPA / UEPA. Fone:633.4761. e-mail: bet2206@aol.com.br

2 Professor Associado do Departamento de Enfermagem Geral e Especializada da EERP-USP.

${ }^{3}$ Doutor da Secção de Apoio Laboratorial EERP-USP.
} 


\section{INTRODUÇÃO}

No Brasil, o câncer constitui a terceira causa de morte, ficando apenas atrás das doenças circulatórias e das causas externas, sendo a segunda causa de morte por doença. Segundo o Sistema de Informação sobre Mortalidade do Ministério da Saúde (SIM/MS), o Instituto Nacional de Câncer (INCA) estimou, para o ano de 2002, 337.535 casos novos e 122.600 óbitos por câncer em todo o país. Para os homens, esperam-se $165.895(49 \%)$ casos novos e 66.060 (54\%) óbitos; no caso das mulheres, 171.640 (51\%) casos novos e 56.540 (46\%) óbitos. Verificamos que apesar do número maior de casos novos entre as mulheres, a mortalidade por câncer é menor do que entre os homens ${ }^{2-3}$.

A Associates in Otolaryngology Head \& Neck Surgery estima que mais de 55.000 americanos desenvolverão câncer de cabeça e pescoço, e aproximadamente 13.000 morrerão no ano de $2003^{4}$. O Sistema de Informação sobre Mortalidade do Ministério da Saúde, no Brasil, informou que a ocorrência de óbitos por câncer de laringe no ano de 1999 foi de 2.575 (2.25\%). É importante enfatizar que o câncer de laringe é o mais comum na região da cabeça e pescoço, sendo que aproximadamente $25 \%$ dos tumores malignos atingem essa região, e que, de todas as neoplasias malignas, eles somam $2 \%$. Os números mostram que dois terços dessas neoplasias aparecem na corda vocal verdadeira e um terço acomete a laringe supraglótica².

Os fatores de risco relacionados ao câncer de laringe, de acordo com a literatura e os estudos desenvolvidos na área, são: abuso da voz, laringite crônica, tabagismo, etilismo e os hábitos precários de higiene bucal. Há, ainda os fatores ocupacionais, o contato com fibras têxteis, níquel, pó de madeira e asbesto ${ }^{1-2,4}$.

Os principais tratamentos para o câncer de cabeça e pescoço indicam a cirurgia, radioterapia, quimioterapia, hormonioterapia e imunoterapia ${ }^{1}$. Eles são empregados com objetivo de cura, mas em determinadas situações tornam-se tratamentos paliativos, podendo ser indicados sozinhos ou em combinação, definido segundo o tipo de tumor, extensão, condição clínica e as preferências do paciente. O correto diagnóstico e o estadiamento do tumor são elementos imprescindíveis no processo de tomada de decisão acerca do tipo de tratamento5.

Atualmente, os enfoques da cirurgia estão voltados para preservação da função da laringe e para os meios eficazes de reconstrução, ou seja, favorecem a ressecção total da neoplasia maligna, com a finalidade de preservar ao máximo a função respiratória, fonatória e esfincteriana ${ }^{6}$. Nos casos de tumores de laringe com estadiamento T3 e T4, em geral os pacientes são submetidos à laringectomia total, intervenção na qual se removem a laringe cartilaginosa, osso hióide e os músculos infra-hióideos. A radioterapia tem sido indicada para os pacientes com comprometimento de apenas uma das cordas vocais ${ }^{7}$.

As complicações pós-operatórias da laringectomia total são: hematoma; obstrução respiratória; edema; infecção da ferida cirúrgica; deiscência de sutura; hemorragia; distúrbio da deglutição; estenose da traqueostomia e formação de fístula faringocutânea, tendo esta última um alto índice de ocorrência. Os dois estudos retrospectivos sobre as complicações pósoperatórias dos pacientes submetidos à laringectomia total associada à dissecção radical do pescoço identificaram a fístula faringocutânea como a mais freqüente complicação com os percentuais, de $70 \%$ e $30 \%$ respectivamente ${ }^{8-9}$. Em função dessa complicação no pós-operatório, os laringectomizados totais têm a sua alimentação oral adiada, prolongando-se o período de hospitalização e aumentando-se a probabilidade da cirurgia adicional.

A etiologia da fístula faringocutânea é ainda bastante discutida, porém vários fatores de risco são encontrados na literatura e nas investigações, tais como: radioterapia pré-operatória; dissecção radical do pescoço; material de sutura utilizado para reconstrução faríngea; traqueostomia pré-operatória; doenças sistêmicas; estadiamento do tumor; transfusão sangüínea no transoperatório; nível de hemoglobina no pósoperatório; tipo de drenagem do pescoço; infecção da ferida e formação de hematoma ${ }^{15}$. A atuação da enfermeira, nestes casos, é promover a melhoria da qualidade das intervenções de enfermagem direcionadas aos problemas clínicos dos pacientes. Esta responsabilidade requer o desenvolvimento de intervençôes visando ao atendimento individualizado do paciente, procurando guiá-lo para a tomada de decisão na prática clínica ${ }^{10}$.

A partir do que foi exposto, evidenciamos a importância da obtenção de dados por meio da coleta, de diagnóstico de enfermagem, planejamento, implementação e avaliação das intervenções de enfermagem no período perioperatório pelo qual passa o portador de câncer de laringe. Para desenvolver estas açōes, é necessário também que as enfermeiras conheçam o que é a fístula faringocutânea, um problema freqüente no período pós-operatório de laringectomia total.

Assim, o objetivo desse estudo é analisar a freqüência dos fatores de risco para o desenvolvimento da fístula faringocutânea encontrados na literatura. 


\section{METODOLOGIA}

Trata-se de um estudo do tipo descritivo, no qual os fenômenos foram descritos ou a relação entre as variáveis é examinada. A pesquisa descritiva tem por finalidade observar, descrever e explorar os aspectos de uma situação ou seja, descrever "com exatidão" os fatos ou fenômenos de uma determinada realidade ${ }^{11,13}$.

Selecionamos as publicações referentes aos fatores de risco que favorecem a formação da fístula faringocutânea, após laringectomia total, nos Bancos de Dados do PUBMED e MEDLINE, bases de dados da literatura internacional da área médica e biomédica, produzida pela National Library of Medicine (NLM), no período de março de 2001 a março de 2002.

Utilizamos para o levantamento dos artigos os seguintes descritores: (larynx cancer OR larynx neoplasia) AND (total laryngectomy) OR (pharyngocutaneous fistula) AND (risk factors) OR (frequency), excluindo-se aqueles que não abordavam os fatores de risco e a freqüência de fístula faringocutânea após laringectomia total.

Investigamos 20 artigos indexados nos bancos de dados acima mencionados e a amostra foi composta apenas de 10 artigos, todos obtidos na íntegra.

\section{RESULTADOS}

A fístula faringocutânea é a complicação mais comum do pós-operatório de laringectomia total e o seu desenvolvimento pode complicar o período pósoperatório, duplicando a média de hospitalização e conseqüentemente os custos da mesma.

$\mathrm{Na}$ revisão de literatura obtivemos a freqüência e os fatores de risco para o desenvolvimento da fístula faringocutânea, após laringectomia total, os quais estão descritos na Tabela 1.
Como podemos observar na Tabela 1 , as maiores freqüências foram identificadas em dois estudos retrospectivos. O primeiro mostrou que dos 357 pacientes submetidos à laringectomia total, 84(23\%) desenvolveram a fístula faringocutânea, tendo estes recebido radioterapia pré-operatória. Os autores constaram que o surgimento da fístula ocorreu com tempo médio de 7 dias após a cirurgia ${ }^{17}$.

Em outra investigação com 310 pacientes submetidos à laringectomia total, observou-se que $28(9 \%)$ pacientes desenvolveram a fístula faringocutânea, num período de 6 a 11 dias. $\mathrm{E}$ os fatores de risco relacionados à crescente taxa de formação de fístula foram a idade do paciente, hipertensão arterial e o nível de hemoglobina baixo $^{16}$.

$\mathrm{Na}$ Tabela 2 apresentamos a incidência da fístula faringocutânea em pacientes submetidos a radioterapia pré-operatória antes da laringectomia total, que variou de 5,0 a 46,0\% nos estudos de número1,2,3,4,5,6 e 8. No estudo de número 7 os autores enfatizam que as fístulas surgiram após um episódio de vômito que foi relatado nos registros médico e de enfermagem, sendo o tempo médio em que ocorreu o vômito de 7,5 dias (oscilou de 1 a10 dias), e o tempo entre o episódio de vômito e o diagnóstico da fístula de 0,4 a 1,2 dias. No estudo de número 9, os autores examinaram o papel dos estudos radiográficos contrastados no período pósoperatório mediato em pacientes laringectomizados totais para identificar precocemente o desenvolvimento da fístula faringocutânea. No estudo número 10 os pacientes incluídos se submeteram a laringectomia neartotal, no entanto, os tumores não foram irradiados previamente.

Nos outros oito estudos restantes, o surgimento da fístula faringocutânea ocorreu num período médio de 5

Tabela 1. Freqüência e Porcentagem de Fístulas Faringocutâneas encontradas pelos autores dos artigos analisados. Ribeirão Preto (SP), 2003.

\begin{tabular}{|c|c|c|c|c|c|}
\hline Autor & Pacientes (n) & Freqüência (f) & $\begin{array}{c}\text { Fístulas } \\
(\%)\end{array}$ & $\begin{array}{l}\text { Tempo de } \\
\text { Internação } \\
\text { (dias) }\end{array}$ & $\begin{array}{c}\text { Tempo de } \\
\text { Cicatrização } \\
\text { (dias) }\end{array}$ \\
\hline 1.Cheen; Siow, $1999^{14}$ & 69 & 11 & 15,9 & $18-42$ & - \\
\hline 2.Zinis et al, $1999^{15}$ & 246 & 40 & 16,0 & - & 39 a 46 \\
\hline 3.Papazoglou et al, $1994^{16}$ & 310 & 28 & 9,0 & - & 39 \\
\hline 4.McCombe; Jones, $1992^{17}$ & 357 & 84 & 23,0 & - & 28 a 112 \\
\hline 5.Friedman et al $1999^{18}$ & 138 & 28 & 20,3 & 8 a 22 & 9,2 \\
\hline 6.Ikiz et al. $2000^{19}$ & 92 & 08 & 8,7 & 43,38 & 14 a 56 \\
\hline 7.Tomkinson et al. $1996^{20}$ & 50 & 08 & 16,0 & - & - \\
\hline 8.Fradis et al. $1995^{21}$ & 56 & 07 & 12,5 & 25,5 & 14 \\
\hline 9.Moses et al. $1993^{22}$ & 132 & 28 & 21,0 & 44 & - \\
\hline 10.Gavilán et al. $1996^{23}$ & 46 & 23 & 15,0 & 37 & - \\
\hline
\end{tabular}


Tabela 2. Incidência de Fístulas Faringocutâneas em Pacientes Submetidos à Radioterapia Pré-Operatória.Ribeirão Preto (SP), 2003.

\begin{tabular}{|c|c|c|c|c|}
\hline \multirow{2}{*}{ Autores } & \multirow{2}{*}{ Participantes } & \multirow{2}{*}{$\begin{array}{l}\text { Radioterapia } \\
\text { Pré-operatória }\end{array}$} & \multicolumn{2}{|c|}{ Fístula } \\
\hline & & & $\mathrm{N}$ & $\%$ \\
\hline 1.Cheen; Siow, $1999^{14}$ & 69 & 14 & 07 & 5,0 \\
\hline $2 . Z$ inis et al, $1999^{15}$ & 246 & 37 & 05 & 15,0 \\
\hline 3.Papazoglou et al, $1994^{16}$ & 310 & 125 & 18 & 14,0 \\
\hline 4.McCombe; Jones, $1992^{17}$ & 357 & 357 & 74 & 21,0 \\
\hline 5.Friedman et al $1999^{18}$ & 138 & 138 & 34 & 25,0 \\
\hline 6.Ikiz et al. $2000^{19}$ & 92 & 06 & 06 & 40,0 \\
\hline 7.Tomkinson et al. $1996^{20}$ & 50 & - & 06 & 75,0 \\
\hline 8.Fradis et al. $1995^{21}$ & 56 & 26 & 07 & 46,0 \\
\hline 9.Moses et al. $1993^{22}$ & 132 & \multicolumn{3}{|c|}{ Não consta dados. } \\
\hline 10.Gavilán et al. $1996^{23}$ & 46 & \multicolumn{3}{|c|}{ Pacientes não irradiados previamente. } \\
\hline
\end{tabular}

a 104 dias, onde identificamos os seguintes fatores de risco: radioterapia pré-operatória; insuficiência cardíaca crônica; hipertensão arterial; insuficiência hepática; nível de hemoglobina baixo; febre $\left(38.5^{\circ} \mathrm{C}\right)$; estadiamento do tumor; dissecção radical do pescoço e vômito ${ }^{14-23}$.

Verificamos que a ocorrência da fístula faringocutânea após laringectomia total requer do enfermeiro planejamento da assistência de enfermagem para o laringectomizado total. E para realização desse procedimento é necessário que este profissional possua conhecimento técnico-científico da fisiologia de formação da fístula faringocutânea aliado à busca de evidências para tomada de decisão, tendo em vista a definição das prescrições de enfermagem.

Ressaltamos que os pacientes com câncer de laringe apresentam, muitas vezes, problemas colaborativos ou comorbidades, que influenciam a evolução de todo o tratamento, constituindo-se fatores de risco para a formação da fístula faringocutânea, após laringectomia total.

A ocorrência de fístulas faringocutâneas em pacientes laringectomizados totais variou de 8,7 a $23,0 \%$, e o tempo de internação hospitalar foi de 12 a 46 dias, ou seja, uma média de internação igual a 28 dias $^{15-23}$.

Outro aspecto importante a ser enfatizado diz respeito ao período de cicatrização da fístula, que foi de 11,1 a 61 dias, perfazendo um tempo médio de cicatrização de 36 dias. Ao considerarmos a média de internação hospitalar de 28 dias e o tempo médio de cicatrização das fístulas faringocutâneas de $36^{15-23}$, percebemos que houve aumento significativo de cuidados intensivos de enfermagem direcionados a esses pacientes durante todo o período de internação, pois este problema de difícil tratamento exige maior investimento financeiro para manutenção do atendimento hospitalar.

Identificamos a adoção de duas modalidades de tratamento da fístula faringocutânea: o conservador e o cirúrgico. O tratamento conservador utilizado pelos autores deste trabalho constituiu-se de curativo oclusivo da fístula faríngea; cuidados de limpeza com a ferida; uso da sonda nasogástrica; higiene oral; antibioticoterapia; remoção de todo tecido necrosado e nutrição adequada. Já o tratamento cirúrgico exige o desbridamento cirúrgico e posterior fechamento com os retalhos miocutâneo do músculo peitoral maior e deltopeitoral.

\section{DISCUSSÃO}

Apesar do enfoque técnico, consideramos imprescindíveis a realização destes cuidados para atender às necessidades básicas do laringectomizado total. Para tanto, é essencial que a enfermeira estabeleça um relacionamento interpessoal com o paciente, a fim de lhe explicar todas as etapas e procedimentos do tratamento da fístula faringocutânea, tornando-o um participante ativo do seu tratamento. $\mathrm{O}$ investimento da equipe de enfermagem no desenvolvimento desses cuidados poderá prevenir ou minimizar a necessidade do tratamento cirúrgico para a fístula faringocutânea.

A identificação dos fatores de risco para o desenvolvimento da fístula no pré-operatório é imprescindível para o planejamento das intervenções de enfermagem. Para tanto, a enfermagem deverá elaborar um instrumento de coleta de dados direcionado aos pacientes portadores de câncer de cabeça e pescoço, com flexibilidade para introdução de informações relevantes sobre o paciente entrevistado, a fim de que a assistência de enfermagem seja individualizada no atendimento das suas necessidades específicas, no período perioperatório.

Assim, depreendemos que a enfermeira tem papel preponderante no tratamento da fístula faringocutânea 
do laringectomizado total, principalmente na intervenção conservadora, pois é o profissional que, basicamente, presta cuidados de enfermagem diretos e intensivos ao paciente no tratamento da ferida e se mantém alerta quanto aos fatores intervenientes da evolução da fístula faringocutânea. Então, cabe à enfermeira aplicar os princípios científicos na avaliação rigorosa da condição geral do paciente e na evolução cicatricial da fístula faringocutânea, indicando produtos nãomedicamentosos para o tratamento da lesão. Também é a enfermeira quem deve avaliar os cuidados higiênicos da cavidade bucal, da traqueostomia, da sonda nasogástrica e supervisionar a administração da antibioticoterapia.

A fístula faringocutânea, após laringectomia total, é a complicação mais grave e freqüente nos laringectomizados totais, contribuindo não só para aumentar o período de internação hospitalar como também elevar os recursos humanos e materiais.

Desse modo, depreendemos que o conhecimento técnico-científico da enfermeira sobre a fístula faringocutânea é imprescindível para o planejamento da assistência de enfermagem no atendimento das necessidades humanas básicas do laringectomizado total.

A qualidade das intervenções de enfermagem está diretamente relacionada à habilidade de raciocínio clínico e técnico e ao relacionamento interpessoal da enfermeira na abordagem do laringectomizado total em todo o período de tratamento.

\section{CONCLUSÃO}

Esta investigação apresenta uma freqüência alta de fístulas faringocutâneas após a laringectomia total. Os fatores de risco relacionados à crescente taxa de formação de fístulas, apontados pelos autores, foram a radioterapia pré-operatória; dissecção radical do pescoço; tipo de material de sutura utilizado; traqueostomia pré-operatória; doenças sistêmicas; estadiamento do tumor; transfusão sangüínea no transoperatório; tipo de drenagem do pescoço; infecção da ferida. O surgimento da fístula faringocutânea nos pacientes ocorreu num período médio de 5 a 104 dias, com ocorrência de 8,7 a 23,0 \% e tempo de internação hospitalar de 12 a 46 dias, com média igual a 28 dias. Quanto ao período de cicatrização da fístula, este foi de 11,1 a 61 dias, perfazendo média de 36 dias.

Julgamos que os resultados deste estudo são importantes para que a enfermeira possa planejar e implementar uma assistência adequada ao paciente portador de fístula faringocutânea, após laringectomia total.

\section{REFERÊNCIAS BIBLIOGRÁFICAS}

1. American Cancer Society. Cancer Facts \& Figures. Available from: URL:http://www.cancer.org./statistic/96ctt/ tacts.html

2. Instituto Nacional de Câncer (Brasil). Epidemiologia. Disponível em: http://www.inca.org.br

3. Ministério da Saúde. Secretaria Executiva DATASUS (Brasil). Sistema de informações sobre mortalidade. Disponível em: http://www.datasus.gov.br

4. Associates in Otolaryngology Head \& Neck Surgery. Head neck cancer. Available from: URL:www.sciencedirect.com/ science/journal/01960709

5. Forastiere A, Koch W, Tnotti A, Sidransk D. Head and neck cancer. N Engl J Med 2001;345(26):1890-900.

6. Lofiego JL. Laringectomia: avaliação e terapia fonoaudiólogo. 1a ed. Rio de Janeiro (RJ): Revinter; 1994.

7. Noranha MJR, Dias FL. Câncer da laringe: uma abordagem multidisciplinar. 1a ed. Rio de Janeiro de Janeiro (RJ): Revinter; 1998.

8. Sawada NO, Zago MMF, Galvão MC, Ferreira E, Barichello E. Complicaçōes pós-operatórias nas laringectomias totais: um estudo retrospectivo. Rev Bras Cancerol 1998;44(1): 35-41.

9. Magrin J, Kowalski LP. Complicaçōes pós-operatórias em pacientes submetidos a esvaziamento cervical radical bilateral simultâneo. Acta Oncol Bras 1996;16(1):3-11.

10. Sidani S, Braden CJ. Evaluating nursing interventions: a theory-driven approach. Thousand Oaks (CA): Sage; 1998.

11. Nieswiadomy RM. Foundations of nursing research. 3rd ed. Stamford (Connecticut): Appleton \& Lange; 1998.

12. Triviños AN. Introdução à pesquisa em ciências sociais: a pesquisa qualitativa em educação. 4a ed. São Paulo (SP): Altas; 1995.

13. Polit DF, Hungler BP. Fundamentos de pesquisa em enfermagem. 3a ed. Porto Alegre (RS): Artes Médicas; 1995.

14. Cheen N, Siow JK. Pharyngocutaneous fistula after laryngectomy: incidence, predisposing factors and outcome. Singapore Med J 1999;40(3):130-2.

15. Zinis LOR, Ferrari L, Tomenzoli D, Premoli G, Parrinello G, Nicolai P. Postlaryngectomy pharyngocutaneous fistula: incidence, predisposing factors, and therapy. Head Neck 1999;21(2):131-8.

16. Papazoglou G, Terzakis G, Doundoulakis G, Dokianakis G. Pharyngocutaneous fistula after total laryngectomy: incidence, cause, and treatment. Ann Otol Rhinol Laryngol 1994;130:801-5.

17. McCombe AW, Jones AS. Radiotherapy and complications of laryngectomy. J Laryngol Otol 1993;107:130-2.

18. Friedman M, Venkatesan TK, Yakovlev A, Lim JW, Tanyeri HM, Caldarelli DD. Early detection and treatment of postoperative pharyngocutaneous fistula. Otolaryngol Head 
Neck Surg 1999;121(4):378-80.

19. Ikiz AO, Uça M, Güneri EA, Erdag TK, Sütays S. Pharyngocutaneous fistula and total laryngectomy: possible predisposing factors, with emphasis on pharyngeal myotomy. J Laryngol Otol 2000;114:768-71.

20. Tomkinson A, Shone GR, Dingle A, Roblin DG, Quine S. Pharyngocutaneous fistula following total laryngectomy and post-operative vomiting. Clin Otolaryngol 1996;21:369-70.
21. Fradis M, Podoshin L, David JB. Post-laryngectomy pharyngocutaneous fistula-a still unresolved problem. J Laryngol Otol 1995;109:221-4.

22. Moses BL, Eisele DW, Jones B. Radiologic assessment of the early postoperative total-laryngectomy patient. Laryngoscope 1993;103:1157-60.

23. Gavilán J, Prim P, Herranz J, Rabanal I. Seepck results and complications of near-total laryngectomy. Ann Otol Rhinol Laryngol 1996;105:729-33. 